\title{
Energy-Efficient Multihop Cooperative MISO Transmission with Optimal Hop Distance in Wireless Ad Hoc Networks
}

\author{
Jun Zhang, Li Fei, Qiang Gao, Xiao-Hong Peng
}

\begin{abstract}
In this paper, we investigate the hop distance optimization problem in ad hoc networks where cooperative multiinput-single-output (MISO) is adopted to improve the energy efficiency of the network. We first establish the energy model of multihop cooperative MISO transmission. Based on the model, the energy consumption per bit of the network with high node density is minimized numerically by finding an optimal hop distance, and, to get the global minimum energy consumption, both hop distance and the number of cooperating nodes around each relay node for multihop transmission are jointly optimized. We also compare the performance between multihop cooperative MISO transmission and single-input-single-output (SISO) transmission, under the same network condition (high node density). We show that cooperative MISO transmission could be energyinefficient compared with SISO transmission when the path-loss exponent becomes high. We then extend our investigation to the networks with varied node densities and show the effectiveness of the joint optimization method in this scenario using simulation results. It is shown that the optimal results depend on network conditions such as node density and path-loss exponent, and the simulation results are closely matched to those obtained using the numerical models for high node density cases.
\end{abstract}

Index Terms-Cooperative MISO, energy efficiency, hop distance, wireless ad hoc networks.

\section{INTRODUCTION}

$\mathbf{E}$ NERGY efficiency is a key performance considered in the design of wireless ad hoc networks, where nodes are usually powered by batteries with limited capacity and lifetime and deployed in potentially harsh scenarios. When two nodes in a wireless ad hoc network are not in range of each other, the communication between them will rely on multihop transmission. In such a case, hop distance is an important factor that affects the energy consumption of end-toend multihop transmission between the source and destination nodes in the network. Large hop distance increases the energy consumption of a single hop but decreases the number of hops, whilst short hop distance has exact reverse effects. Thus finding an optimal hop distance that minimizes the total energy

Manuscript received December 13, 2010; revised May 14, 2011; accepted July 14, 2011. The associate editor coordinating the review of this paper and approving it for publication was S. Valaee.

J. Zhang, L. Fei, and Q. Gao (corresponding author) are with the School of Electronic and Information Engineering, Beihang University, and National Key Laboratory of CNS/ATM, Beijing 100191, P.R. China (e-mail: \{zhangjun, feili\}@ee.buaa.edu.cn, gaoqiang@buaa.edu.cn).

X.-H. Peng is with the School of Engineering and Applied Science, Aston University, Birmingham B4 7ET, United Kingdom (e-mail: xh.peng@aston.ac.uk).

Digital Object Identifier 10.1109/TWC.2011.081011.102210 consumed within the entire transmission path plays a vital role in deploying energy-efficient and sustainable wireless ad hoc networks in real-world environments.

There have been many research results published on the optimization of hop distance (or transmission range) with different focuses. Some results give the relations between hop distance and other system or network parameters such as the number of transmission source, network topology [1], path-loss exponent, node density [2], and bit-error-rate (BER) requirement [3]; while the others show how to achieve the optimal hop distance and optimal transmitting power when transmission error is taken into account [4] or realistic channel models such as the Rayleigh fading and Lognormal fading models are considered [5]. In addition, some works investigate the optimal transmission range alongside specific routing protocols such as the greedy forwarding routing protocol [6]. However, all of these results are obtained for the wireless networks that use single-input-single-output (SISO) transmission.

Recently, cooperative multi-input-multi-output (MIMO) technique and its variations, e.g. cooperative multi-inputsingle-output (MISO), are adopted in wireless multihop ad hoc networks to improve energy efficiency over SISO systems by exploiting the spatial diversity created through cooperative communication [7]-[8]. Many studies in this research area have been trying to exploit fully the advantages of cooperative MIMO (or its variations) in energy saving by proposing specific schemes in routing [9], routing with power control [10][12], routing with link scheduling [13], and optimizing the number of cooperating nodes per hop to minimize the end-toend energy consumption while satisfying the required outage probability [14]. On top of these schemes, hop distance is another key parameter that can be optimized to further improve energy efficiency of multihop cooperative transmission.

The optimization problem will then become more complex for multihop cooperative MIMO (or its variations) transmission compared with SISO transmission. On the one hand, the spatial diversity exploited by cooperative MIMO can reduce the transmission energy under a given BER; thus a larger hop distance may be chosen to decrease the number of hops. On the other hand, extra energy overhead is needed for the source or relay node to transmit data to the local cooperating nodes before over-hop cooperative transmission. This means that apart from the hop distance, the distribution or the number of cooperating nodes also affects energy consumption for local as well as over-hop transmissions. Therefore, finding the optimal 
hop distance for maximizing the overall energy efficiency for cooperative MIMO (or its variations) transmission presents a challenging problem in this research area. The solution to this problem, however, is previously unknown, to the best of our knowledge.

In this paper, we present our investigation over the impacts of both hop distance and the number of cooperating nodes on the energy consumption of end-to-end multihop cooperative MISO transmission. This investigation leads to a joint optimization approach that considers both hop distance and the number of cooperating nodes in the process of energy efficiency maximization under different network conditions. For simplicity, we consider the routing schemes as adopted in [9], [13], and [14], where a relay node and its neighboring nodes (not relay nodes) cooperatively transmit data to its next relay node. However, other schemes as used in [10]-[12], where a relay node and its previous relay nodes in the routing path cooperatively transmit data to its next relay node, are not considered in this paper. In this work, we first deal with the joint optimization problem heuristically in two stages in a high-density ad hoc network where the relay nodes on the route of multihop transmission are always available at the desired locations when they are needed. When the average distance between any two adjacent nodes is much less than the hop distance, the network can be considered highly dense. We then propose a cooperative MISO multihop transmission scheme, and extend our investigation to networks without a constraint on node density through simulation. The simulation results show that the gap between the numerically optimized hop distance and the one obtained through simulation is within $10 \%$ until the node density is reduced to $10^{-5}$ nodes $/ \mathrm{m}^{2}$ for the case where the path-loss exponent is two (or to $10^{-3}$ nodes $/ \mathrm{m}^{2}$ when the path-loss exponent is four). This means that the numerical optimization results obtained under high node density can be still valid in these ranges of node density. When the node density is further reduced the gap will increase noticeably. We also analyze and compare the energy performance between cooperative MISO and SISO transmission systems, to reveal the conditions under which cooperative MISO can be more energy-efficient than SISO and the other way around in the context of multihop transmission.

The rest of this paper is organized as follows. In Section II, the energy model of multihop cooperative MISO transmission is established. In Section III, hop distance and number of cooperating nodes are jointly optimized to minimize energy consumption per bit in a high-density network. Numerical results and performance analysis are given in Section IV. A cooperative MISO multihop transmission scheme and related simulation results obtained under general network conditions are given in Section V. Finally, Section VI summarizes the outcomes of this paper.

\section{ENERGy Model}

We consider a wireless ad hoc network where each node is equipped with one antenna. Suppose that a source $S$ intends to transmit some data packets to a destination $D$. If $D$ is out of the transmission range of $S$ (or for the sake of energy efficiency), relay nodes between $S$ and $D$ will be used to forward the data packets to the destination. To save

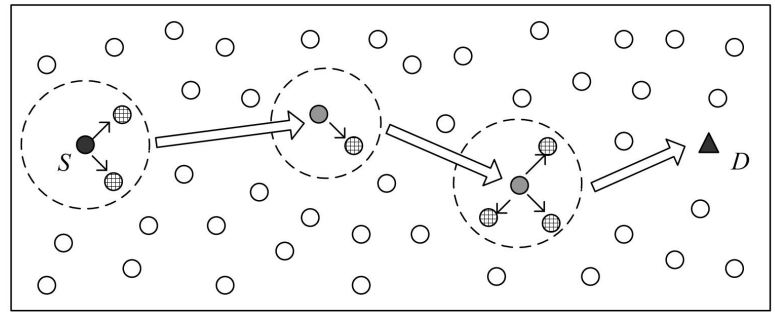

$\bigcirc$ Relay node $\oplus$ Cooperating node $\rightarrow$ Broadcasting $\Longrightarrow$ Cooperative transmission

Fig. 1: System overview of multihop cooperative MISO transmission.

energy, the relay nodes (or source) and their neighboring nodes can cooperatively transmit these packets to the next relay node or the final destination, as shown in Fig. 1. In this case, the transmitting nodes and the receiving node construct a cooperative MISO system. Specifically, the transmission process of each hop consists of two phases.

- Phase 1 (Broadcasting phase). The relay node (or source in the first hop) broadcasts the data to the nodes around it. The nodes which receive the broadcasted data and participate in cooperative transmission in the next phase are called cooperating nodes.

- Phase 2 (Cooperative transmission phase). The relay node (or source in the first hop) and the cooperating nodes concurrently transmit the data to the next relay node using the distributed orthogonal space-time block code (STBC).

We assume a flat Rayleigh fading channel with additive white Gaussian noise (AWGN) on top of $\alpha$ th-power path loss, where $\alpha \geq 2$ is the path-loss exponent. The fading between all transmitting and receiving nodes are assumed to be independent and identically distributed and to be constant during the transmission of each packet. Multilevel quadrature amplitude modulation (MQAM) is deployed for data transmission. No error correction code (ECC) blocks are included in the transmitted data.

To calculate the energy consumption per bit of data transmission from source to destination, both circuit energy consumption and transmission energy for data broadcasting and cooperative transmission are included in our energy model as in [7]-[8]. We do not consider the energy consumed by overhearing, as in [1]-[4], nor the extra energy consumption due to retransmission, as in [1], [2], [11], and [12]. As routing and medium access control (MAC) protocols are not discussed in this paper, the related signaling energy is not considered, either.

According to the transmission process described above, the energy consumption per bit of one hop $E_{h}$ consists of two components: energy consumption in the broadcasting phase $E_{b p}$ and energy consumption in the cooperative transmission phase $E_{c p}$, i.e.,

$$
E_{h}=E_{b p}+E_{c p}
$$

$E_{b p}$ is given by

$$
E_{b p}=E_{b p}^{c}+E_{b p}^{t}
$$

where $E_{b p}^{c}$ is the circuit energy consumed by the relay node for transmission and by the cooperating nodes for reception, and 
$E_{b p}^{t}$ is the transmission energy for the relay node to broadcast its data.

The circuit energy consumption $E_{b p}^{c}$ is given by

$$
E_{b p}^{c}=\frac{P_{c t}}{R}+N \cdot \frac{P_{c r}}{R}
$$

where $P_{c t}$ is the transmitter circuit power, $P_{c r}$ is the receiver circuit power, $N$ is the number of cooperating nodes, and $R$ is the bit rate of broadcasting, which is given by $R=b \cdot B$ with $b$ the constellation size (bits per symbol) and $B$ the modulation bandwidth.

The transmission energy of the relay node $E_{b p}^{t}$ is given by

$$
E_{b p}^{t}=G \cdot r^{\alpha} \cdot \bar{E}_{b, b p}
$$

where $r$ is the radius of the neighboring area in which $N$ cooperating nodes lies, $\bar{E}_{b, b p}$ is the required average energy per bit at the cooperating node for the given BER requirement. $G$ is a constant and given by $G=\frac{\xi}{\eta} \cdot \frac{(4 \pi)^{2}}{G_{t} G_{r} \lambda^{2}} \cdot M_{l} \cdot N_{f}$, as adopted in [2] and [8] as well, where $\xi=3 \cdot\left(\frac{2^{b / 2}-1}{2^{b / 2}+1}\right)$ is the peak-to-average ratio (PAR) and $\eta$ is the drain efficiency of the RF power amplifier, $G_{t}$ is the transmitter antenna gain, $G_{r}$ is the receiver antenna gain, $\lambda$ is the carrier wavelength, $M_{l}$ is the link margin compensating hardware process variations and other additive background noise or interference, and $N_{f}$ is the receiver noise figure.

Assuming that nodes are distributed in the network with density $\rho$, the radius $r$ of the neighboring area in which $N$ cooperating nodes lies is give by

$$
r=\sqrt{\frac{N+1}{\rho \cdot \pi}}
$$

The average energy per bit at the cooperating node $\bar{E}_{b, b p}$ is determined by the required BER. For MQAM, the average BER is given by

$$
\bar{P}_{b, b p} \approx \int_{0}^{+\infty} C_{1} \cdot Q\left(\sqrt{C_{2} \cdot \gamma_{b p}}\right) \cdot f\left(\gamma_{b p}\right) d \gamma_{b p}
$$

where $Q(\cdot)$ denotes the Q-function, $C_{1}$ and $C_{2}$ are the constants determined by constellation size [7]. $\gamma_{b p}$ is the instantaneous signal-to-noise ratio (SNR) at the receiver and has an exponential distribution as

$$
f\left(\gamma_{b p}\right)=\frac{1}{\bar{\gamma}_{b p}} \cdot e^{-\frac{\gamma_{b p}}{\bar{\gamma}_{b p}}}
$$

$\bar{\gamma}_{b p}$ is given by

$$
\bar{\gamma}_{b p}=\frac{\bar{E}_{b, b p}}{N_{0}}
$$

where $N_{0}$ is the single-sided thermal noise power spectral density.

Referring to (1) again, the second term $E_{c p}$ is given by

$$
E_{c p}=E_{c p}^{c}+E_{c p}^{t}
$$

where $E_{c p}^{c}$ is the circuit energy consumed at the transmitting side by the relay node and the cooperating nodes and at the receiving side by the next relay node, and $E_{c p}^{t}$ is the transmission energy per bit for cooperative data transmission.
Let $R_{s}$ denote the spatial rate of $\mathrm{STBC}$, the bit rate of cooperative transmission is given by $R \cdot R_{s}$ [15], thus we have

$$
E_{c p}^{c}=(N+1) \cdot \frac{P_{c t}}{R \cdot R_{s}}+\frac{P_{c r}}{R \cdot R_{s}}
$$

The extra circuit energy consumed due to STBC coding and decoding, which is not comparable with the energy consumed by radio frequency (RF) circuit blocks, is neglected in our case. Therefore, $P_{c t}$ and $P_{c r}$ in (10) are the same as that in (3), respectively.

The transmission energy per bit for cooperative data transmission $E_{c p}^{t}$ is given by

$$
E_{c p}^{t}=G \cdot d^{\alpha} \cdot \bar{E}_{b, c p}
$$

where $\bar{E}_{b, c p}$ is the required average energy per bit at the next relay node for a given BER requirement, $d$ is the hop distance, i.e. the distance between two nearby relay nodes. Equation (11) holds only when the distances from the relay node and its cooperating nodes to the next relay node are the same. This is a reasonable assumption when the hop distance is much larger than the radius of the relay node's neighborhood, which is the case for most our results. When the hop distance becomes comparable with the neighborhood radius, (11) could still approximately hold since the perceived power difference between the signals from any two transmitting nodes is not significant in most cases [16].

The average energy per bit at the next relay node $\bar{E}_{b, c p}$ is determined by the required BER. Similar to the broadcasting phase, the average BER required in the cooperative transmission phase $\bar{P}_{b, c p}$ is given by

$$
\bar{P}_{b, c p} \approx \int_{0}^{+\infty} C_{1} \cdot Q\left(\sqrt{C_{2} \cdot \gamma_{c p}}\right) \cdot f\left(\gamma_{c p}\right) d \gamma_{c p}
$$

$\gamma_{c p}$ is the instantaneous SNR at the next relay node and has a central chi-square distribution with $2(N+1)$ degrees of freedom as [17]

$$
f\left(\gamma_{c p}\right)=\frac{1}{\Gamma(N+1) \cdot \bar{\gamma}_{c p}^{N+1}} \cdot \gamma_{c p}^{N} \cdot e^{-\frac{\gamma_{c p}}{\bar{\gamma}_{c p}}}
$$

where $\Gamma(\cdot)$ denotes the Gamma function, and $\bar{\gamma}_{c p}$ is given by

$$
\bar{\gamma}_{c p}=\frac{\bar{E}_{b, c p}}{(N+1) \cdot N_{0}}
$$

Combining (1)-(5) and (9)-(11) and considering the fact that $\bar{E}_{b, c p}$ is a function of $N$, the energy consumption per bit of one hop $E_{h}$ can be obtained as

$$
E_{h}=g_{1}(N)+g_{2}(N) \cdot d^{\alpha}
$$

where $g_{1}(N)=\frac{P_{c t}}{R}+N \cdot \frac{P_{c r}}{R}+G \cdot\left(\sqrt{\frac{N+1}{\rho \cdot \pi}}\right)^{\alpha} \cdot \bar{E}_{b, b p}+(N+$ 1) $\cdot \frac{P_{c t}}{R \cdot R_{s}}+\frac{P_{c r}}{R \cdot R_{s}}$, and $g_{2}(N)=G \cdot \bar{E}_{b, c p}$.

Summing the energy consumption per bit of all the hops, we obtain the energy consumption per bit from source to destination $E_{b i t}$ as

$$
E_{b i t}=\sum_{k=1}^{K} E_{h}^{(k)}=\sum_{k=1}^{K}\left[g_{1}\left(N_{k}\right)+g_{2}\left(N_{k}\right) \cdot d_{k}^{\alpha}\right]
$$

where $E_{h}^{(k)}$ is the energy consumption per bit of the $k$ th hop, $d_{k}$ and $N_{k}$ are the hop distance and number of cooperating nodes of the $k$ th hop respectively, and $K$ is the total number of hops between source and destination. 


\section{ENERGY CONSUMPTION MINIMIZATION}

In this section, the hop distance and the number of cooperating nodes are jointly optimized to minimize the energy consumption per bit from the source to the destination based on the energy model established in the last section. The optimization problem can be formulated as

$$
\begin{array}{cl}
\min _{K, d_{k}, N_{k}, k=1,2, \ldots, K} & E_{b i t}=\sum_{k=1}^{K}\left[g_{1}\left(N_{k}\right)+g_{2}\left(N_{k}\right) \cdot d_{k}^{\alpha}\right] \\
\text { s.t. } & \sum_{k=1}^{K} d_{k} \geq L \\
& d_{k}>0, k=1,2, \ldots, K \\
& N_{k} \text { is a positive integer, } k=1,2, \ldots, K \\
& K \text { is a positive integer }
\end{array}
$$

where $L$ is the distance between the source and the destination.

This is a joint optimization problem of $2 K+1$ variables, i.e. $d_{k}, N_{k}(k=1,2, \ldots, K)$, and $K$. As $K$ increases, a significant number of variables need to be processed and the problem becomes highly complex. To reduce the complexity level, a high-density ad hoc network is assumed. Under the high-density assumption, the data will be transmitted through a linear route to achieve the minimum energy consumption. As a result, the first constraint in (17) is changed to $\sum_{k=1}^{K} d_{k}=L$. Consequently, the following proposition can be obtained.

Proposition 1: Under high node density, given the distance between the source and the destination $L$ and the number of hops $K$, if all the hop distances are equal (i.e. $\forall k: d_{k}=\frac{L}{K}$ ) and the numbers of cooperating nodes $N_{k}$ are the same for all hops, the minimum energy consumption per bit can be achieved.

\section{Proof: See Appendix A.}

Therefore, setting hop distances identical for all hops is sufficient for achieving the minimum energy consumption. It is the trend that the hop distances will become very close to each other for all hops when the minimum energy consumption is intended to be achieved since all the hops are homogeneous.

Setting $d_{k}=d$ and $N_{k}=N(k=1,2, \ldots, K)$, we have the optimization problem as

$$
\begin{array}{ll}
\min _{d, N} & E_{b i t}=\frac{L}{d} \cdot\left[g_{1}(N)+g_{2}(N) \cdot d^{\alpha}\right] \\
\text { s.t. } & d \in\left\{L, \frac{L}{2}, \frac{L}{3}, \ldots\right\} \\
& N \text { is a positive integer }
\end{array}
$$

To further simplify the optimization problem, we loosen the corresponding constraint and let $d$ be a real variable, so that the problem becomes

$$
\begin{array}{ll}
\min _{d, N} & E_{b i t}=\frac{L}{d} \cdot\left[g_{1}(N)+g_{2}(N) \cdot d^{\alpha}\right] \\
\text { s.t. } & 0<d \leq L \\
& N \text { is a positive integer }
\end{array}
$$

The optimization problem specified in (19) can be solved heuristically through two stages, first minimizing over hop distance $d$ and then minimizing over the number of cooperating nodes $N$, based on [18].

Stage 1: The hop distance $d$ is optimized to minimize the energy consumption per bit given the number of cooperating nodes $N$. At this stage, the optimization problem is reduced to

$$
\begin{array}{cl}
\min _{d} & E_{b i t}=\frac{L}{d} \cdot\left[g_{1}(N)+g_{2}(N) \cdot d^{\alpha}\right] \\
\text { s.t. } & 0<d \leq L
\end{array}
$$

The convexity of $E_{b i t}$ is shown as follows.

Proposition 2: Given the number of cooperating nodes $N$, $E_{b i t}$ in (20) is a convex function of $d$.

Proof: See Appendix B.

Taking the first-order derivative of $E_{b i t}$ with respect to $d$ and setting it to zero, we obtain the optimal hop distance $d^{*}$ by solving this equation, as

$$
d^{*}=\sqrt[\alpha]{\frac{g_{1}(N)}{(\alpha-1) \cdot g_{2}(N)}}
$$

Correspondingly, the miniminzed energy consumption per bit $E_{b i t}^{*}$ is

$$
E_{b i t}^{*}=\frac{\alpha \cdot L}{\alpha-1} \cdot \sqrt[\alpha]{(\alpha-1) \cdot\left[g_{1}(N)\right]^{\alpha-1} \cdot g_{2}(N)}
$$

We call $d^{*}$ and $E_{b i t}^{*}$ the local optimal hop distance and local minimum energy consumption per bit, respectively.

Stage 2: The number of cooperating nodes $N$ is optimized to further minimize the local minimum energy consumption $E_{b i t}^{*}$, which can be formulated as

$$
\begin{array}{ll}
\min _{N} & E_{b i t}^{*}=\frac{\alpha \cdot L}{\alpha-1} \cdot \sqrt[\alpha]{(\alpha-1) \cdot\left[g_{1}(N)\right]^{\alpha-1} \cdot g_{2}(N)} \\
\text { s.t. } & N \text { is a positive integer }
\end{array}
$$

and then the global minimum energy consumption per bit $E_{b i t}^{o p t}$ is obtained as $E_{b i t}^{o p t}=\min _{N} E_{b i t}^{*}$.

As $g_{2}(N)$ is an implicit function of $N$, it is not easy to derive the closed form of the optimal number of cooperating nodes $N^{o p t}$ from (23). Using the brute-force search method $N^{\text {opt }}$ can be determined, and consequently the optimal hop distance $d^{o p t}$ can be obtained by substituting $N^{o p t}$ into (21). According to [17] and [19], we set the number of cooperating nodes that the source or relay node searches for to be 10 . In the following proposition, we show that $d^{o p t}$ and $N^{o p t}$ do not vary with the overall distance $L$.

Proposition 3: Under the assumption of high node density and letting $d$ be a real variable, the optimal hop distance $d^{o p t}$ and the optimal number of cooperating nodes $N^{o p t}$ are independent of the distance between the source and the destination $L$.

Proof: See Appendix C.

Note that if the distance $L$ from source to destination is shorter than or equal to the broadcast radius $r$ used, it is not necessary to carry out the cooperative transmission specified in Phase 2. Therefore, we have $d^{\text {opt }}=r$ and $N^{o p t}=0$ if $L \leq r$

Similar to multihop cooperative MISO transmission, the optimal hop distance that minimizes the energy consumption of multihop SISO transmission, $d_{S I S O}^{o p t}$, can also be obtained under the assumption of high node density [1], i.e.

$$
d_{S I S O}^{o p t}=\sqrt[\alpha]{\frac{e_{1}}{(\alpha-1) \cdot e_{2}}}
$$

where $e_{1}=\frac{P_{c t}+P_{c r}}{R}$, and $e_{2}=G \cdot \bar{E}_{b, S I S O} \cdot \bar{E}_{b, S I S O}$ denotes the average energy per bit for the required BER of SISO. Correspondingly, the minimum energy consumption per bit of multihop SISO transmission $E_{S I S O}^{o p t}$ is given by

$$
E_{S I S O}^{o p t}=\frac{\alpha \cdot L}{\alpha-1} \cdot \sqrt[\alpha]{(\alpha-1) \cdot e_{1}^{\alpha-1} \cdot e_{2}}
$$


TABLE I: Circuit and system parameters

\begin{tabular}{l|l}
\hline$P_{c t}=150 \mathrm{~mW}$ & $P_{c r}=100 \mathrm{~mW}$ \\
$B=10 \mathrm{kHz}$ & $b=2$ \\
$\eta=0.35$ & $G_{t} G_{r}=5 \mathrm{dBi}$ \\
$M_{L}=40 \mathrm{~dB}$ & $N_{0}=-171 \mathrm{dBm} / \mathrm{Hz}$ \\
$N_{f}=10 \mathrm{~dB}$ & $R_{s}=1 / 2$ \\
$\lambda=0.12 \mathrm{~m}$ & $\bar{P}_{b, b p}=\bar{P}_{b, c p}=10^{-4}$ \\
$\alpha=2$ & $\rho=1$ nodes $/ \mathrm{m}^{2}$ \\
$L=5000 \mathrm{~m}$ & \\
\hline
\end{tabular}

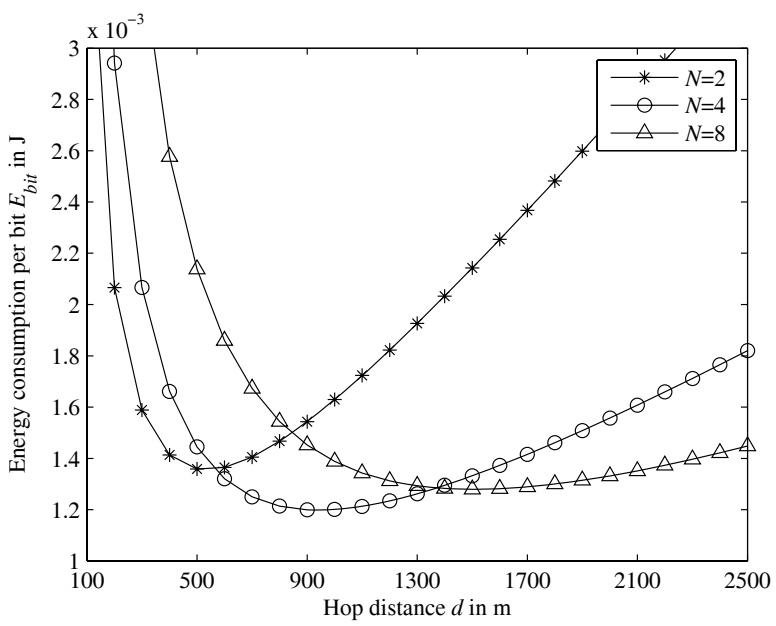

Fig. 2: Energy consumption per bit over hop distance under different numbers of cooperating nodes for $\rho=1$.

\section{NUMERICAL RESUlTS}

The numerical results of the joint optimization of the hop distance and the number of cooperating nodes under high node density are presented in this section. The circuit and system parameters, in line with other research works, are summarized in Table I. The constellation size $b$ is chosen to be 2 , thus we have $C_{1}=1$ and $C_{2}=2$ according to [7]. The spatial rate $R_{s}$ is set to be $1 / 2$, as a rate- $1 / 2$ orthogonal STBC can be used regardless of the number of antenna elements (cooperating nodes) based on the generalized complex orthogonal design [15]. The node density $\rho$ is chosen to be 1 nodes $/ \mathrm{m}^{2}$ thus the average distance between two adjacent nodes is about $1 \mathrm{~m}$, while the optimal hop distance obtained ranges from decades to hundreds meters, thus the network can be considered to be highly dense.

The optimization results for cooperative MISO obtained using (19) are shown in Fig. 2, in which the energy consumption per bit $E_{b i t}$ is plotted against the hop distance $d$ for different numbers of cooperating nodes. It can be seen that for a given number of cooperating nodes the local optimal hop distance $d^{*}$ can be determined to minimize the energy consumption per bit. Clearly, energy saving through the hop distance optimization is shown to be significant. It is also shown that the local optimal hop distance varies with the number of cooperating nodes, indicating the possibility of finding $N^{\text {opt }}$, which leads to the global minimum energy consumption $E_{b i t}^{o p t}$.

In Fig. 3, the local minimum energy consumption per bit $E_{b i t}^{*}$ is plotted against the number of cooperating nodes $N$. It is shown that the optimal number of cooperating nodes $N^{\text {opt }}$ can be determined to minimize $E_{b i t}^{*}$. Given the circuit and

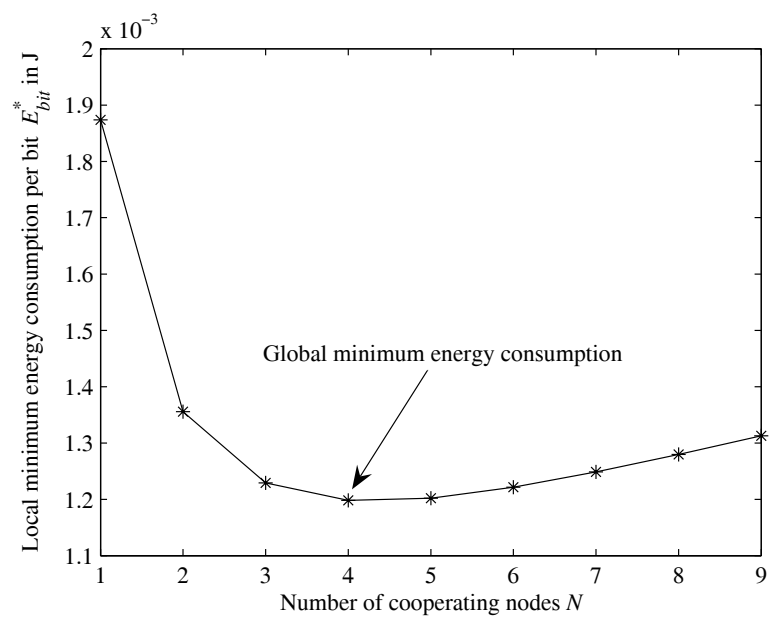

Fig. 3: Local minimum energy consumption per bit over the number of cooperating nodes.

system parameters in Table I, the global minimum energy consumption per bit $E_{b i t}^{o p t}$ obtained by the joint optimization is $1.19 \times 10^{-3} \mathrm{~J}$. The corresponding optimal number of cooperating nodes $N^{o p t}$ is 4, and the optimal hop distance $d^{o p t}$ is 939.2 $\mathrm{m}$. For the same circuit and system parameters, the optimal hop distance for multihop cooperative MISO transmission is much larger than that of SISO transmission given by (24). For example, the optimal hop distance of SISO transmission is only $25.2 \mathrm{~m}$ for the parameters given in Table I. This can be explained by the fact that the spatial diversity exploited by cooperative MISO will reduce the transmission energy under a given BER, thus a larger hop distance is preferred for cooperative MISO than SISO for energy-efficient data transmission.

Note that when different circuit and system parameters are chosen, the values of the above optimal results vary accordingly. The BER requirement is an important parameter, and the impact of BER requirement on cooperative MISO transmission in multihop networks has been studied in [12]. For instance, when the required BER is set to be $10^{-3}$ and other parameters are the same as listed in Table I, the optimal hop distance, the optimal number of cooperating nodes and the global minimum energy consumption per bit are 1077.2 $\mathrm{m}, 3$ and $8.59 \times 10^{-4} \mathrm{~J}$, respectively.

As the path-loss exponent, which reflects the condition of transmission channel, increases, the optimal hop distances of both multihop cooperative MISO and SISO transmission, i.e. $d^{o p t}$ and $d_{S I S O}^{\text {opt }}$, will decrease, as shown in Fig. 4. This reaction is due to the increased path loss that forces the system to choose a shorter hop distance in order to meet the BER requirement at the receiver with a fixed transmitting power. It is also shown that $d^{o p t}$ changes faster than $d_{S I S O}^{\text {opt }}$, since the energy consumption of one hop transmission in cooperative MISO is more sensitive to the change of path loss conditions than that of SISO transmission. The optimal hop distance is shown in the logarithmic scale.

In Fig. 5, the minimum energy consumption per bit against the path-loss exponent is shown for both multihop cooperative MISO and SISO transmission, in terms of $E_{b i t}^{o p t}$ versus $E_{S I S O}^{o p t}$. 


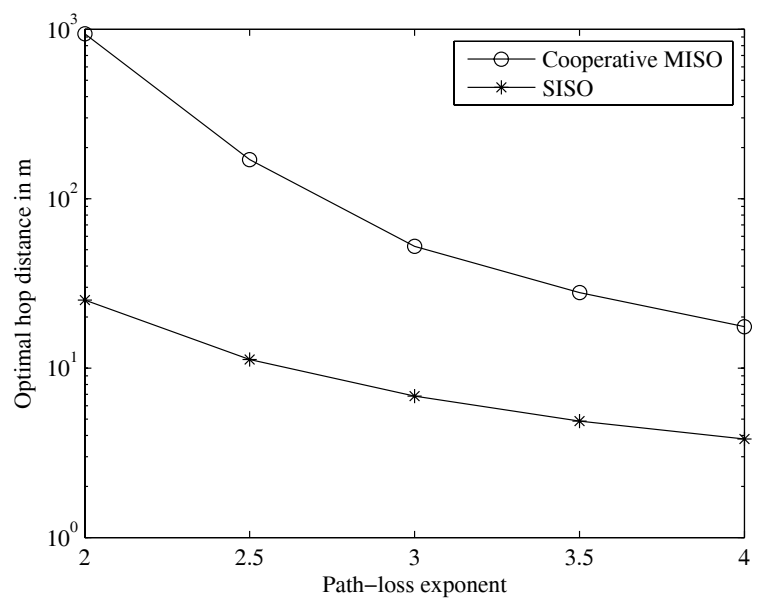

Fig. 4: Optimal hop distance comparison between cooperative MISO and SISO for different path-loss exponents.

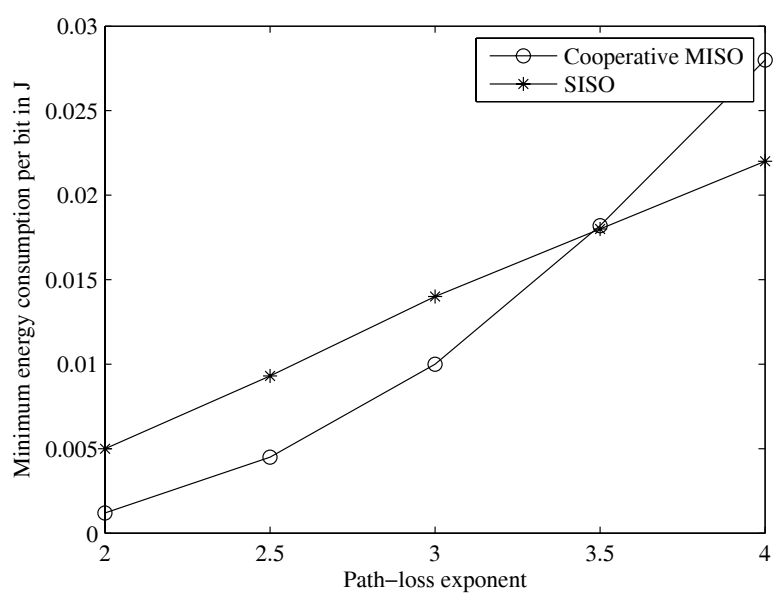

Fig. 5: Energy efficiency comparison between cooperative MISO and SISO for different path-loss exponents.

It can be seen that when the path-loss exponent is 2, which is the case of low path-loss, cooperative MISO transmission consumes about $80 \%$ less energy than SISO transmission. However, in the case of high path loss $(\alpha>3.5)$ cooperative MISO transmission could consume more energy than SISO transmission.

To explain the performance of $E_{b i t}^{o p t}$ versus $E_{S I S O}^{o p t}$ given in Fig. 5, detailed energy consumption elements that contributed to both $E_{b i t}^{o p t}$ and $E_{S I S O}^{o p t}$ are shown in Fig. 6, including transmission and circuit energy consumption and energy costs at different phases of the cooperative MISO scheme. As it can be seen, with low path-loss exponents, both transmission and circuit energy consumption of Phase 2 for cooperative MISO transmission are much less than those for SISO transmission. In addition, the energy consumption of Phase 1 for cooperative MISO is also very small because when the path-loss exponent is low the number of hops needed for multihop cooperative MISO transmission is much less than that for multihop SISO transmission. As the path-loss exponent increases, the number of hops for MISO transmission increases at a faster pace than SISO transmission, causing both transmission and circuit

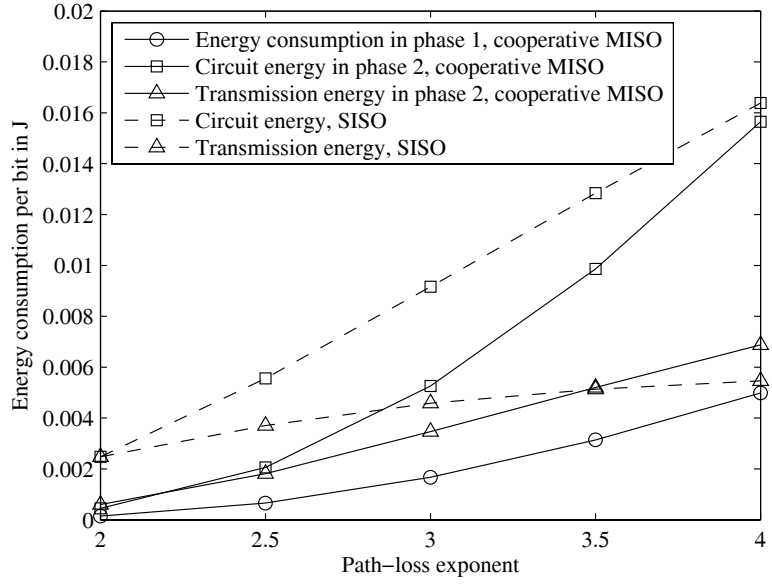

Fig. 6: Energy consumption comparison between cooperative MISO and SISO.

energy consumptions of Phase 2 to increase considerably and the energy consumption of Phase 1 to become more noticeable. As a result, $E_{b i t}^{o p t}$ could exceed $E_{S I S O}^{o p t}$ when the channel condition is poor, i.e. with high path-loss exponents, as shown in Fig. 5.

Note that the circuit energy in Phase 2 with cooperative MISO is increasing with a higher slope with increasing pathloss exponent $\alpha$ than that for transmission energy as shown in Fig. 6. When the path-loss exponent increases from 2 to 4 the optimal hop distance decreases sharply from 939.2 $\mathrm{m}$ to $17.4 \mathrm{~m}$, as shown in Fig. 4. As transmission energy consumed in Phase 2 depends on both hop distance and link conditions represented by the path-loss exponent, this mixed effect (i.e. decrease in $d^{\text {opt }}$ and increase in $\alpha$ ) will cause the transmission energy to increase slowly overall. Meanwhile, the sharp decrease in hop distance means sharp increase in the number of hops, thus the increase in circuit energy in Phase 2 at a much higher pace than what has happened to transmission energy is expected. This is because the circuit energy consumption is directly related to the number of transmission hops.

\section{A CoOperative Miso Multihop Transmission SCHEME}

In this section, we propose a cooperative MISO multihop transmission scheme that applies to networks without a constraint on node density, and investigate the performance of the joint optimization method discussed above in this scenario through simulation.

\section{A. Scheme Description}

We consider a wireless ad hoc network where node distribution is not necessarily highly dense. Each node is assumed to know its own location and all other nodes' locations in the network. This can be achieved by applying any of existing localization algorithms [20]. Suppose that a source $S$ intends to transmit a data packet to a destination $D$. The multihop transmission scheme proposed is performed as follows. 


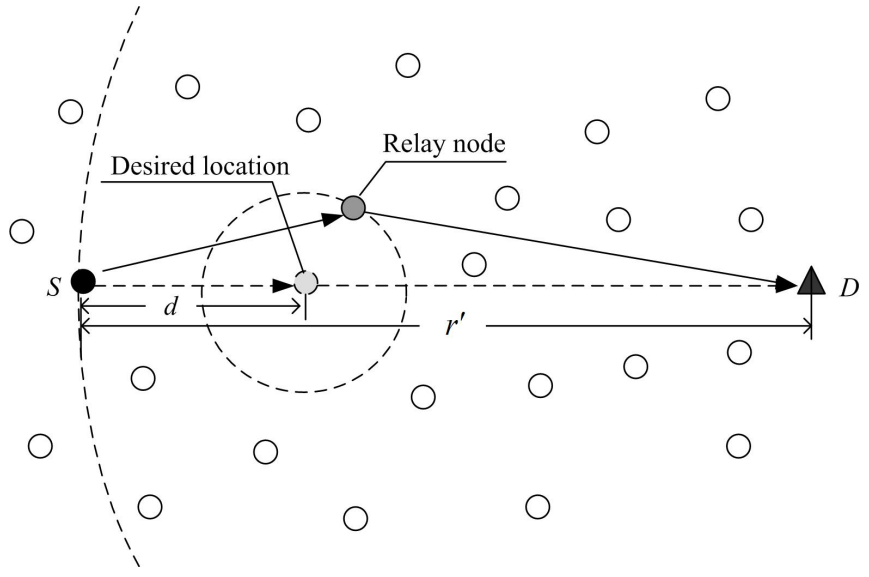

Fig. 7: Illustration of the proposed scheme.

Step 1) Relay node selection. To achieve minimum energy consumption the source prefers to transmit data to the relay node that lies in the linear route between the source and the destination with hop distance $d$. However, in the networks where node density is low, nodes are not always available at exactly the desired locations. In this situation the source has to select the node closest to the desired location to be the relay node instead (cf. Fig. 7). If the node density is extremely low the relay node selected may lie in the opposite direction to the destination. To avoid this situation, the selected relay node should be closer to the destination than the source, thus it must lie within the circle centered at the destination with the radius, $r^{\prime}$, which is made by the direct line between the source and destination, as indicated in Fig. 7. If the destination is closer to source than the desired location, it will be selected as the relay node directly.

Step 2) Cooperating nodes selection. The source selects $N$ closest nodes around it as cooperating nodes. For the cases that the number of close-by nodes is less than $N$, all the nodes that can receive the broadcasted data packets in step 3) will be selected as cooperating nodes.

Step 3) Data packet transmission. The source first broadcasts data packets to the cooperating nodes, and then the source and the cooperating nodes transmit these packets to the selected relay node simultaneously using distributed orthogonal STBC.

Step 4) Next-hop transmission. The selected relay node repeats Step 1)-Step 3) as a new source node to forward the data, until data packets reach the destination.

\section{B. Simulation Results}

The optimal hop distance and optimal number of cooperating nodes that minimize the energy consumption of the proposed transmission scheme are identified through simulation in a general network. In our simulations, the nodes are uniformly distributed with node density $\rho$, and the source and the destination are located at $(0,0)$ and $(5000 \mathrm{~m}, 0)$, respectively, on a two-dimensional plane. All the other parameters used are chosen from Table I except node density $\rho$ and path-loss exponent $\alpha$.

In Fig. 8, the energy consumption per bit is plotted against the hop distance for different numbers of cooperating nodes for $\alpha=2$ and $\rho=0.01$. Similar to in Fig. 2, we can see

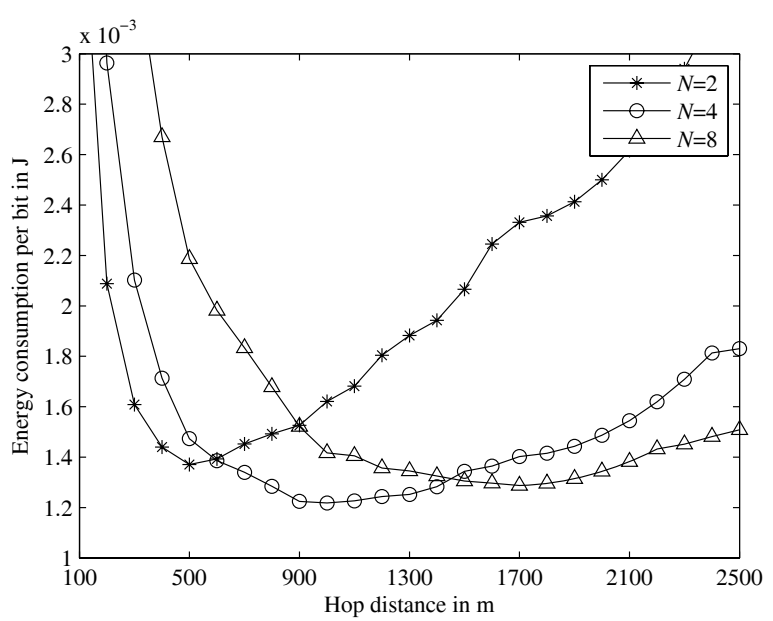

Fig. 8: Energy consumption per bit over hop distance under different numbers of cooperating nodes for $\alpha=2$ and $\rho=$ 0.01 .

that the optimal hop distance exists to achieve the minimum energy consumption for a given number of cooperating nodes. Based on these results, we can also obtain the global minimum energy consumption by identifying the optimal number of cooperating nodes using the same approach as used in Section IV. For the given parameters as applied to Fig. 8, the global minimum energy consumption per bit is $1.22 \times 10^{-3} \mathrm{~J}$, and the corresponding optimal number of cooperating nodes and the optimal hop distance are 4 and $1008 \mathrm{~m}$, respectively.

The optimal hop distance and optimal number of cooperating nodes obtained are the criteria used for the selection of relay nodes and cooperating nodes, respectively, in the proposed cooperative MISO multihop transmission scheme. The real values of hop distance and the number of cooperating nodes used may be different from the optimal ones, especially when the network is less dense. Fig. 9 illustrates the comparison between the optimized multihop cooperative MISO transmission in a high-density network (Fig. 9(a)) and that in a low-density network (Fig. 9(b)). When the network is highly dense, data is transmitted through a linear route. The hop distances and the numbers of cooperating nodes are identical for all hops and they are optimal. However, for the low-density network, the selected relay nodes will drift off the linear route, and the hop distances used may not be identical. Since the number of the close-by nodes around relay nodes may be less than the optimal number of cooperating nodes required, and all close-by nodes will participate in cooperative transmission, the actual number of cooperating nodes may be different at each hop.

The relationships between the optimal hop distance and node density are demonstrated in Fig. 10(a) for cooperative MISO transmission and in Fig. 10(b) for SISO transmission, respectively. In Fig. 10(a), the simulation results show that for the low path-loss exponent $(\alpha=2)$, the optimal hop distance of cooperative MISO transmission is almost unchanged and matches to the numerical result $(\rho=1)$, which is plotted as a baseline for the purpose of comparison, when node density is higher than $10^{-5}$ nodes $/ \mathrm{m}^{2}$. We can also see that the gap 


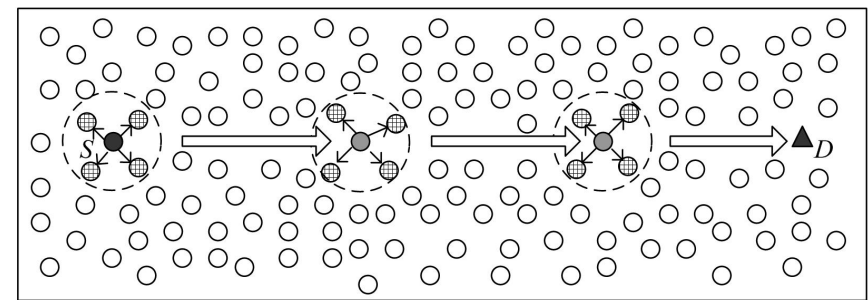

O Relay node $\oplus$ Cooperating node $\rightarrow$ Broadcasting $\Rightarrow$ Cooperative transmission (a) High-density network

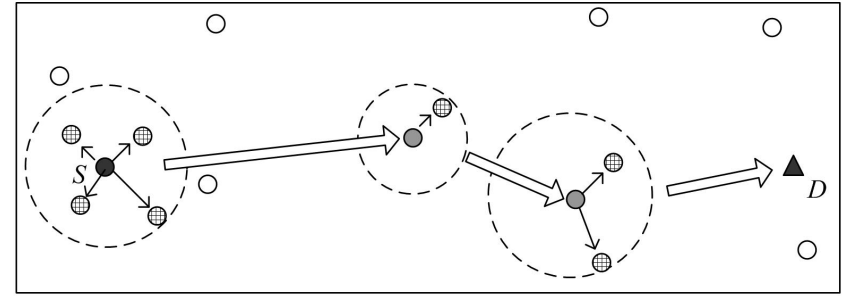

Relay node $\oplus$ Cooperating node $\rightarrow$ Broadcasting $\Rightarrow$ Cooperative transmission (b) Low-density network

Fig. 9: Optimized multihop cooperative MISO transmission comparison between high-density and low-density networks.

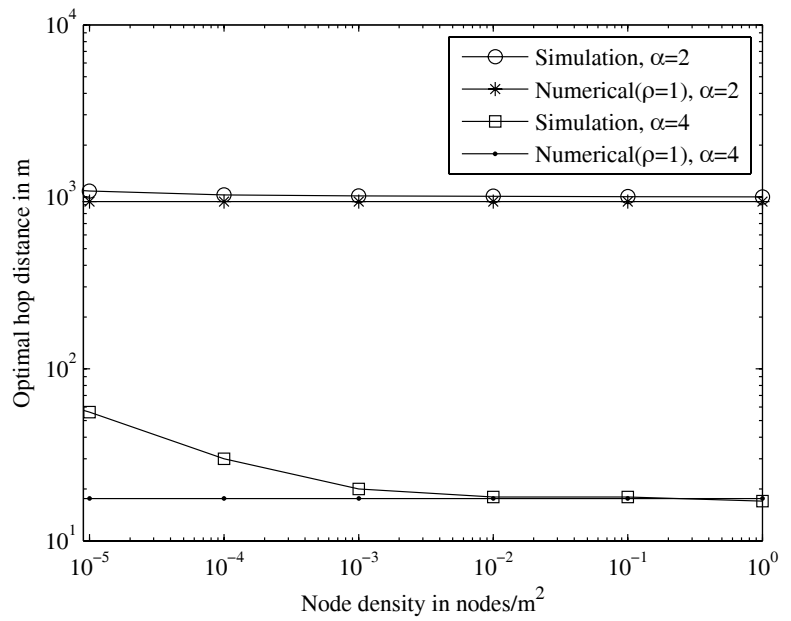

(a) Cooperative MISO

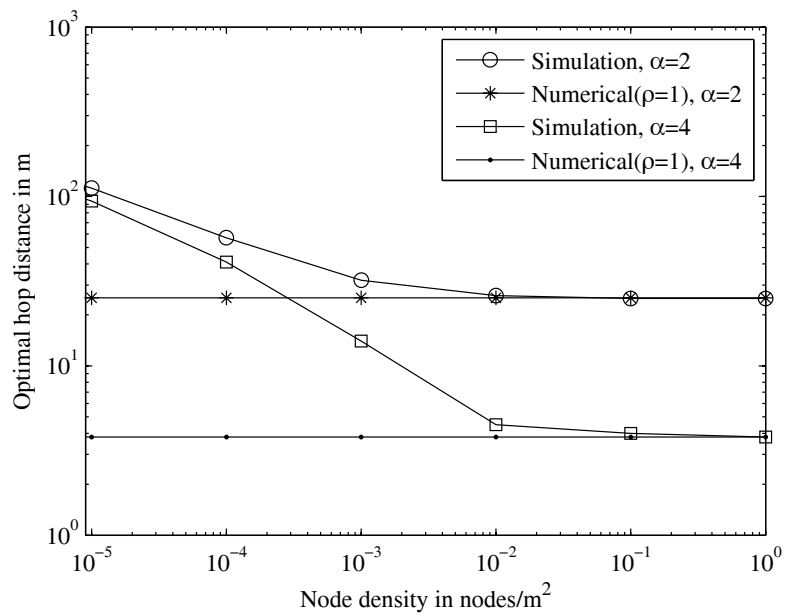

(b) SISO

Fig. 10: Optimal hop distance comparison between simulations for different node densities and numerical results at high node density.

between the simulation and numerical results increases to more than $10 \%$ when the node density is reduced to $10^{-5}$ nodes $/ \mathrm{m}^{2}$. It is also shown that for a higher path-loss exponent $(\alpha=4)$, the optimal hop distance remains nearly constant and the difference from the numerical result $(\rho=1)$ is within $10 \%$ until the node density is reduced to $10^{-3}$ nodes $/ \mathrm{m}^{2}$. Note that as node density decreases to be lower than some values, the hop distance increases noticeably. This implies a decrease in the number of hops. For example, when $\alpha=4$ the number of hops reduces from 288 to 84 as node density reduces from 1 to $10^{-4}$ nodes $/ \mathrm{m}^{2}$.

In Fig. 10(b), it shows that the optimal hop distance in multihop SISO transmission is more sensitive to node density than that in cooperative MISO transmission. We can see that for SISO transmission the optimal hop distance increases noticeably when the node density reduces to $10^{-2}$ nodes $/ \mathrm{m}^{2}$, while for cooperative MISO transmission the noticeable change of optimal hop distance happens at $10^{-3}$ nodes $/ \mathrm{m}^{2}$ for $\alpha=4$ $\left(10^{-5}\right.$ nodes $/ \mathrm{m}^{2}$ for $\left.\alpha=2\right)$. Again, the numerical baseline is also used to compare with the simulation results, showing that the difference between the two methods increases steadily from the point of node density equal to $10^{-2}$ nodes $/ \mathrm{m}^{2}$ and downwards.

The relationship between the optimal number of cooperating nodes and node density for the proposed cooperative MISO transmission scheme is shown in Fig. 11. When $\alpha=2$, the optimal number of cooperating nodes is shown to be independent of node density and the simulation results are the same as the numerical baseline until the node density is reduced to $10^{-5}$ nodes $/ \mathrm{m}^{2}$. When the path-loss exponent is 4 more cooperating nodes are needed when node density decreases from as low as $10^{-2}$ nodes $/ \mathrm{m}^{2}$.

\section{CONCLUSION}

In this paper, we investigate the optimization problem of hop distance in combination with the number of cooperating nodes in an ad hoc network that employs cooperative MISO transmission, in order to improve its energy efficiency. To minimize the energy consumption per bit, hop distance and the number of cooperating nodes are jointly optimized under the condition of high node density, which demonstrates that significant improvements in energy efficiency can be achieved through this optimization. The influence of the path-loss exponent on the optimal results is also investigated for both cooperative MISO and SISO transmissions. It is shown that the maximized energy efficiency of cooperative MISO is higher than that of SISO when the path-loss exponent is low, and this situation is reversed with high path-loss exponents. The joint optimization approach is also applied to a proposed multihop cooperative MISO scheme through simulation in a network 


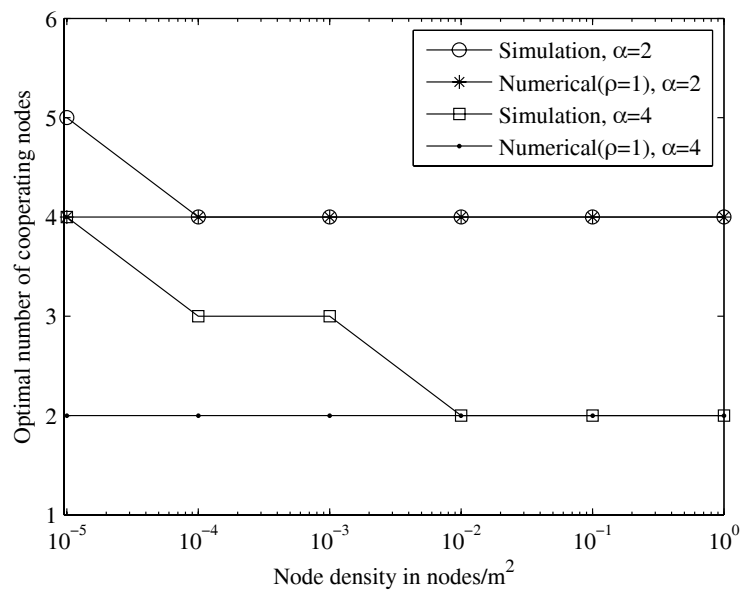

Fig. 11: Optimal number of cooperating nodes comparison between simulations for different node densities and numerical results at high node density.

without the node density constraint. Based on the comparison between simulation and numerical results, it is shown that the numerical optimal results can match the simulation ones when the network is highly dense, and the difference between them in the sparse network is variable depending on the value of the path-loss exponent. Our results can be used as a guideline to the design of energy-efficient communication protocols in wireless ad hoc networks.

Note that in this work we only consider one receiving node at each hop to minimize the combining and decoding complexity at the receiver. A more complex communication process that involves a group of receiving nodes can be investigated by extending our work at this stage.

\section{APPENDIX A}

PROOF OF PROPOSITION 1

To solve the optimization problem in (17) under high node density assumption, i.e. $\sum_{k=1}^{K} d_{k}=L$, we set

$$
F=\sum_{k=1}^{K} E_{h}^{(k)}+\beta \cdot\left(\sum_{k=1}^{K} d_{k}-L\right)
$$

where $\beta \neq 0$ is Lagrange multiplier. Let the number of cooperating nodes take on real values, according to the method of Lagrange multipliers, we obtain

$$
\begin{cases}\frac{\partial E_{h}^{(k)}}{\partial d_{k}}+\beta=0, & k=1,2, \ldots, K \\ \frac{\partial E_{h}^{(k)}}{\partial N_{k}}=0, & k=1,2, \ldots, K \\ \sum_{k=1}^{K} d_{k}=L & \end{cases}
$$

It is shown that $d_{k}$ and $N_{k}$ need satisfy

$$
\left\{\begin{array}{l}
\frac{\partial E_{h}^{(k)}}{\partial d_{k}}=-\beta \\
\frac{\partial E_{h}^{(k)}}{\partial N_{k}}=0
\end{array}\right.
$$

for $k=1,2, \ldots, K$.
Based on the energy model, we can obtain that

$$
\frac{\partial E_{h}^{(k)}}{\partial d_{k}}=\alpha \cdot g_{2}\left(N_{k}\right) \cdot d_{k}^{\alpha-1}
$$

and

$$
\frac{\partial E_{h}^{(k)}}{\partial N_{k}}=\frac{\partial g_{1}\left(N_{k}\right)}{\partial N_{k}}+\frac{\partial g_{2}\left(N_{k}\right)}{\partial N_{k}} \cdot d_{k}^{\alpha}
$$

It can be found that $\frac{\partial E_{h}^{(k)}}{\partial d_{k}}$ and $\frac{\partial E_{h}^{(k)}}{\partial N_{k}}$ vary with $d_{k}$ and $N_{k}$ in the same manner for all the values that $k$ can take on. This implies that, for each value of $k$, (28) has the same set of solutions. Thus setting $d_{k}$ and $N_{k}$ identical for all hops, i.e. $d_{1}=d_{2}=\ldots=d_{K}=L / K$ and $N_{1}=N_{2}=\ldots=N_{K}$, is sufficient for achieving the minimum energy consumption.

\section{APPENDIX B \\ ProOF OF PROPOSITION 2}

Taking the second-order derivative of energy consumption per bit $E_{b i t}$ given in (20) with respect to $d$ for a given $N$, we have

$$
\frac{\partial^{2} E_{b i t}}{\partial d^{2}}=\frac{L}{d^{3}} \cdot\left[(\alpha-1) \cdot(\alpha-2) \cdot g_{2}(N) \cdot d^{\alpha}+2 \cdot g_{1}(N)\right]
$$

Note that $\alpha \geq 2, g_{1}(N)>0, g_{2}(N)>0, L>0$, and $d>0$. As a result, $\frac{\partial^{2} E_{b i t}}{\partial d^{2}}>0$. Therefore energy consumption per bit $E_{b i t}$ given in (20) is a convex function of hop distance $d$ for a given $N$.

\section{APPENDIX C \\ PROOF OF PROPOSITION 3}

We first prove that the optimal number of cooperating nodes $N^{o p t}$ is independent of the distance between source and destination $L . N^{o p t}$ can be obtained by solving the optimization problem given in (23). If the number of cooperating nodes $N$ is considered as a continuous variable, the problem becomes a convex optimization problem.

Taking the first-order derivative of $E_{b i t}^{*}$ in (23) with respect to $N$, we have

$$
\begin{aligned}
\frac{d E_{b i t}^{*}}{d N}= & L \cdot\left\{(\alpha-1) \cdot\left[g_{1}(N)\right]^{\alpha-1} \cdot g_{2}(N)\right\}^{\frac{1-\alpha}{\alpha}} \\
& \cdot\left\{(\alpha-1) \cdot\left[g_{1}(N)\right]^{\alpha-2} \cdot \frac{d g_{1}(N)}{d N} \cdot g_{2}(N)\right. \\
& \left.+\left[g_{1}(N)\right]^{\alpha-1} \cdot \frac{d g_{2}(N)}{d N}\right\}
\end{aligned}
$$

Setting $\frac{d E_{b i t}^{*}}{d N}$ equal to zero, we have

$$
\begin{aligned}
& \left\{(\alpha-1) \cdot\left[g_{1}(N)\right]^{\alpha-1} \cdot g_{2}(N)\right\}^{\frac{1-\alpha}{\alpha}} \\
& \cdot\left\{(\alpha-1) \cdot\left[g_{1}(N)\right]^{\alpha-2} \cdot \frac{d g_{1}(N)}{d N} \cdot g_{2}(N)\right. \\
& \left.+\left[g_{1}(N)\right]^{\alpha-1} \cdot \frac{d g_{2}(N)}{d N}\right\}\left.\right|_{N=N^{o p t}}=0
\end{aligned}
$$

Based on (15), we can find that $g_{1}(N)$ and $g_{2}(N)$ are independent of $L$, thus from (33) it can be easily found that $N^{o p t}$ is independent of $L$.

The optimal hop distance $d^{\text {opt }}$ can be obtained by substituting $N^{o p t}$ into (21). Equation (21) is independent of $L$, thus $d^{\text {opt }}$ is independent of the distance between source and destination $L$. 


\section{ACKNOWLEDGMENT}

This work was supported by the Foundation for Innovative Research Groups of National Natural Science under Grant No. 60921001, and the National Basic Research Program (973 Program) under Grant No. 2011CB707000. The authors would like to express their gratitude and regards to all reviewers for their insightful comments that improved and enhanced this paper.

\section{REFERENCES}

[1] P. Chen, B. O'Dea, and E. Callaway, "Energy efficient system design with optimum transmission range for wireless ad hoc networks," in Proc. IEEE International Conf. Commun., Apr. 2002, vol. 2, pp. 945-952.

[2] J. Deng, Y. S. Han, P.-N. Chen, and P. K. Varshney, "Optimal transmission range for wireless ad hoc networks based on energy efficiency," IEEE Trans. Commun., vol. 55, no. 9, pp. 1772-1782, Sep. 2007.

[3] Q. Chen and M. C. Gursoy, "Energy-efficient modulation design for reliable communication in wireless networks," in Proc. 43rd Annual Conf. Inf. Sciences Syst., Mar. 2009, pp. 811-816.

[4] R. Zhang and J.-M. Gorce, "Optimal transmission range for minimum energy consumption in wireless sensor networks," IEEE Wireless Commun. Netw. Conf., Mar. 2008, pp. 757-762.

[5] W. Feng and J. M. H. Elmirghani, "Energy efficiency in ad-hoc wireless networks with two realistic physical layer models," in Proc. International Conf. Next Generation Mobile Appl., Services Technol., Sep. 2009, pp. 401-406.

[6] Z. Zhang, G. Mao, and B. Anderson, "On the effective energy consumption in wireless sensor networks," IEEE Wireless Commun. Netwo. Conf., Apr. 2010, pp. 1-6.

[7] S. Cui, A. J. Goldsmith, and A. Bahai, "Energy-efficiency of MIMO and cooperative MIMO techniques in sensor networks," IEEE J. Sel. Areas Commun., vol. 22, no. 6, pp. 1089-1098, Aug. 2004

[8] S. K. Jayaweera, "Virtual MIMO-based cooperative communication for energy-constrained wireless sensor networks," IEEE Trans. Wireless Commun., vol. 5, no. 5, pp. 984-989, May 2006.

[9] Y. Yuan, Z. He, and M. Chen, "Virtual MIMO-Based cross-layer design for wireless sensor networks," IEEE Trans. Veh. Technol., vol. 55, no. 3, pp. 856-864, May 2006.

[10] B. Maham, R. Narasimhan, and A. Hjørungnes, "Energy-efficient spacetime coded cooperative routing in multihop wireless networks," in Proc. IEEE Global Telecommun. Conf., Nov. 2009, pp. 1-7.

[11] B. Maham, W. Saad, M. Debbah, Z. Han, and A. Hjørungnes, "Efficient cooperative protocols for general outage-limited multihop wireless networks," in Proc. IEEE International Symp. Personal Indoor Mobile Radio Commun., Sep. 2010, pp. 145-150.

[12] B. Maham and A. Hjørungnes, "Near-optimum power allocation for BER restricted multihop cooperative networks," in Proc. IEEE International Conf. Commun., May 2010, pp. 1-5.

[13] S. Cui and A. J. Goldsmith, "Cross-layer design of energy-constrained networks using cooperative MIMO techniques," Signal Process., vol. 86, pp. 1804-1814, 2006.

[14] A. Aksu and O. Ercetin, "Reliable multi-hop routing with cooperative transmissions in energy-constrained networks," IEEE Trans. Wireless Commun., vol. 7, no. 8, pp. 2861-2865, Aug. 2008.

[15] E. G. Larsson and P. Stoica, Space-Time Block Coding for Wireless Communications. Cambridge University Press, 2003.

[16] G. Jakllari, S. V. Krishnamurthy, M. Faloutsos, P. V. Krishnamurthy, and O. Ercetin, "A cross-layer framework for exploiting virtual MISO links in mobile ad hoc networks," IEEE. Trans. Mobile Comput., vol. 6, no. 6, pp. 579-594, June 2007.
[17] Z. Zhou, S. Zhou, S. Cui, and J. H. Cui, "Energy-efficient cooperative communication in a clustered wireless sensor networks," IEEE Trans. Veh. Technol., vol. 57, no. 6, pp. 3618-3628, Nov. 2008.

[18] S. Boyd and L. Vandenberghe, Convex Optimization. Cambridge University Press, 2004.

[19] S. Haykin and M. Moher, Modern Wireless Communications. Prentice Hall, 2005.

[20] N. Patwari, J. N. Ash, S. Kyperountas, A. O. Hero, R. L. Moses, and N. S. Correal, "Locating the nodes: cooperative localization in wireless sensor networks," IEEE Signal Process. Mag., vol. 22, no. 4, pp. 54-69, June 2005.

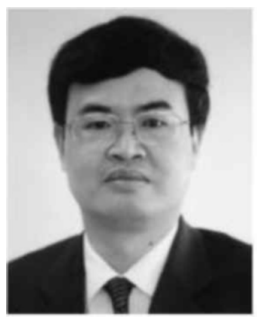

Jun Zhang received the B.S., M.S., and Ph.D. degrees from Beihang University, Beijing, China, in 1987, 1991, and 2001, respectively. He is currently a Professor with the School of Electronic and Information Engineering, Beihang University. His current research interests include integrated networks, network management, and air transportation system.

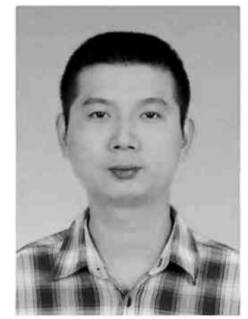

Li Fei was born in Anhui, China, in 1985. He received the B.S. degree in Communication Engineering from North China Electric Power University in 2007 and the M.S. degree in Information and Communication Engineering from Beihang University in 2010. He is currently a Ph.D. student in the School of Electronic and Information Engineering, Beihang University. His major interests are wireless ad hoc networks, MIMO, and cooperative communication.

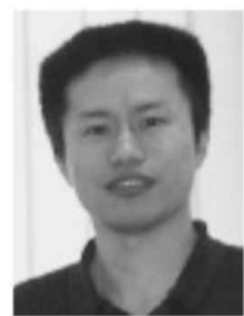

Qiang Gao received his B.S. in Theoretical Physics from Southwest Normal University in 1994, M.S. in Theoretical Physics from Lanzou University in 1997, and Ph.D. degrees in Computer Engineering from Chinese Academy of Science in 2000. He is currently an associate professor with the School of Electronic and Information Engineering, Beihang University, Beijing, P.R.China. His research interests include ad hoc networks, cooperative communication, multimedia networks, and information security.

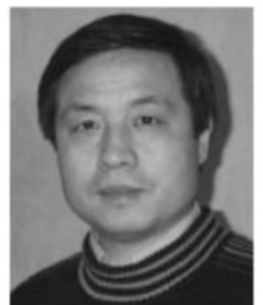

Xiao-Hong Peng received the B.S. and M.S. degrees in electrical engineering from Southwest Jiaotong University, Chengdu, China, in 1982 and 1985, respectively, and the $\mathrm{Ph} . \mathrm{D}$. degree in electronic engineering from the University of Manchester, Manchester, U.K., in 1995.

From 1985 to 1990, he was a Lecturer and Associate Professor with the Department of Computer Science and Engineering, Southwest Jiaotong University. From 1995 to 1997 , he was a Postdoctoral Research Associate with the University of Manchester, and from 1997 to 1998, he was a Research Fellow with the University of York, York, U.K. Between 1998 and 2001, he was with the School of Electronic and Electrical Engineering, South Bank University, London, U.K., as a Lecturer and then as a Senior Lecturer. In 2001, he was with Roke Manor Research, Romsey, U.K., as a Consultant Engineer. In 2002, he joined Aston University, Birmingham, U.K., where he is currently a Senior Lecturer with the Electronic Engineering Subject Group, School of Engineering and Applied Science. His research interests include error-control coding, wireless communications and networking, and distributed networks. 